\title{
THE CONTRACTION MAPPING FOR CONVERGENCE EXPLAINED
}

\section{Saumik Dana ${ }^{1}$}

${ }^{1}$ University of Southern California, Los Angeles, CA 90089

This document is a rendition of the contraction mapping for convergence analysis applied to a staggered solution algorithm in the presence of anisotropy

KEY WORDS: Contraction mapping, convergence, coupled flow and geomechanics, anisotropy

\section{INTRODUCTION}

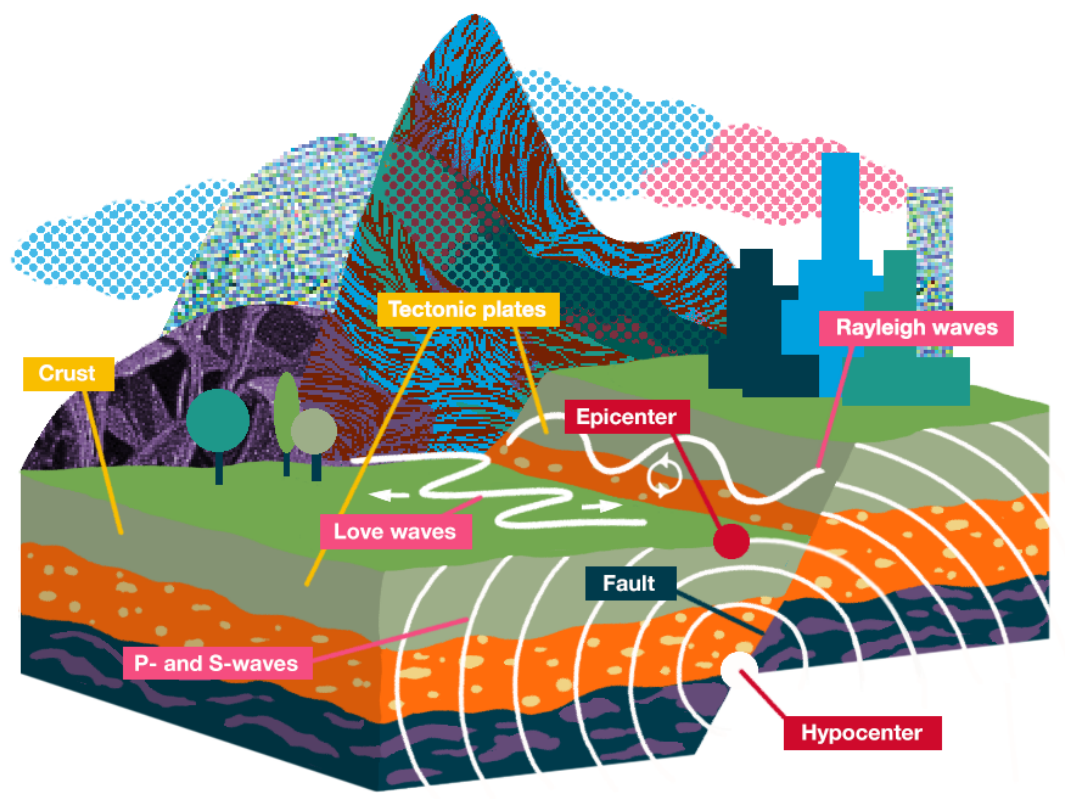

FIG. 1: Source: Caltech Science Exchange

The earthquake cycle, from slow deformation associated with interseismic behavior to rapid deformation associated with earthquake rupture, spans spatial scales ranging from fractions of a meter associated with the size of contact asperities on faults and individual grains to hundreds of kilometers associated with plate boundaries [1]. Similarly, temporal scales range from fractions of a second associated with slip at a point during earthquake rupture to hundreds of years of strain accumulation between earthquakes. In many cases, earthquakes are triggered after pore pressure perturbations activate critically stressed seismogenic faults [1], not just due to natural causes like earth tides [2], rainfall [3], snowfall [4], typhoons [5], but also due to human activity [6]. As faults slip, the induced stress field spawns 


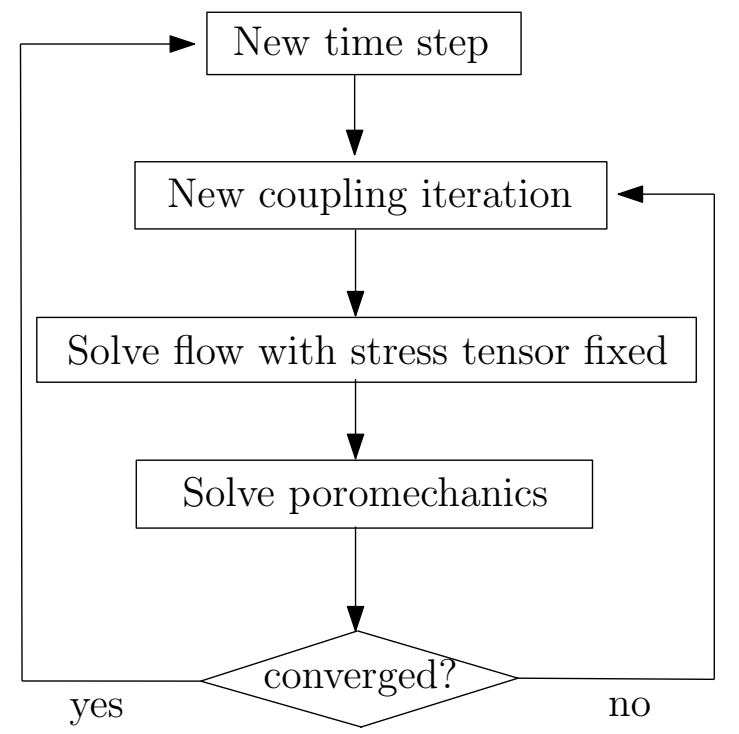

FIG. 2: Fixed stress split iterative scheme for anisotropic poroelasticity with tensor Biot parameter

seismic waves, which travel through and around the earth (see Fig. 1 and are recorded as ground displacement, velocity and/or acceleration time-series data at seismic stations. Understanding the causality between the events leading to fault slip and the recorded seismic data is important for seismic design and monitoring of underground structures [7] and reinforced concrete buildings [8] as well as climate mitigation projects like carbon sequestration [9] and energy technologies like enhanced geothermal systems [10] or oilfield wastewater disposal [11]. Earthquakes lead to losses to the tune of billions of dollars annually in the United States, with the FEMA and USGS pegging the number at \$6.1 billion for the year 2016. The number only includes direct economic losses to buildings, and does not cover damage and losses to critical facilities, transportation and utility lifelines or indirect economic losses. In quasi-static simulations [12-24], the effort is to couple the effects of pore pressure and quasi-static geomechanics, and while that effort is robust when the geomechanical rock is treated as isotropic, it is not robust when the geomechanical rock is treated as anisotropic. In this document, we provide a detailed rendition of the contraction mapping invoked for convergence analysis of [14], which was designed to prove the convergence of the algorithm for anisotropy.

The subject of this document is the extension of the decoupling assumption to the case of anisotropic poroelasticity with tensor Biot parameter. The micromechanical analyses for the case of anisotropic poroelasticity (see [25], [26], [27], [28]) revealed that the modification to the stress applied to the porous solid due to the presence of pore fluid pressure is not hydrostatic, as it is in the case of isotropic poroelasticity (see [29]). Further, unlike in case of isotropic poroelasticity where the solid-fluid coupling parameter is a scalar (see [30], [31], [32], [29]), the coupling parameter in case of anisotropic poroelasticity is a tensor (see [33], [34]). Intuitively, this implies that the decoupling assumption of freezing the hydrostatic part of the stress tensor during the flow solve in every coupling iteration for the case of isotropic poroelasticity requires a generalization for the case of anisotropic poroelasticity. As we shall show in the ensuing convergence analysis, the decoupling assumption of freezing all components of the stress tensor during the flow solve in every coupling iteration does enjoy theoretical convergence for the case of anisotropic poroelasticity with tensor Biot parameter. After that, we shall proceed to use the Mandel's problem analytical solution (see [35]) for the transversely isotropic case to show that the decoupling assumption does enjoy numerical convergence as well.

\subsection{Preliminaries}

The inner product of two second order tensors $\mathbf{S}$ and $\mathbf{T}$ is given by (see [36])

$$
\mathbf{S}: \mathbf{T}=S_{i j} T_{i j} \quad(i, j=1,2,3)
$$


A fourth order tensor is a linear transformation of a second order tensor to a second order tensor in the following manner (see [36])

$$
\mathbb{P} \mathbf{S}=\mathbf{T} \rightarrow \mathbb{P}_{i j k l} S_{k l}=T_{i j} \quad(i, j, k, l=1,2,3)
$$

\section{FLOW MODEL}

The fluid mass conservation equation (1) in the presence of deformable porous medium with the Darcy law (2) and linear pressure dependence of density (3) with boundary conditions (4) and initial conditions (5) is

$$
\begin{aligned}
& \frac{\partial \zeta}{\partial t}+\nabla \cdot \mathbf{z}=q \\
& \mathbf{z}=-\frac{\mathbf{K}}{\mu}(\nabla p-\rho \mathbf{g})=-\mathbf{K}(\nabla p-\rho \mathbf{g}) \\
& \rho=\rho_{0}\left(1+c\left(p-p_{0}\right)\right) \\
& p=g \text { on } \Gamma_{D}^{f} \times(0, T], \mathbf{z} \cdot \mathbf{n}=0 \text { on } \Gamma_{N}^{f} \times(0, T] \\
& p(\mathbf{x}, 0)=p_{0}(\mathbf{x}), \rho(\mathbf{x}, 0)=\rho_{0}(\mathbf{x}), \phi(\mathbf{x}, 0)=\phi_{0}(\mathbf{x}) \quad(\forall \mathbf{x} \in \Omega)
\end{aligned}
$$

where $p: \Omega \times(0, T] \rightarrow \mathbb{R}$ is the fluid pressure, $\mathbf{z}: \Omega \times(0, T] \rightarrow \mathbb{R}^{3}$ is the fluid flux, $\bar{\epsilon}$ is the volumetric strain, $\zeta \equiv \frac{1}{M} p+\alpha \bar{\epsilon}$ is the fluid content, $\Gamma_{D}^{f}$ is the Dirichlet boundary, $\mathbf{n}$ is the unit outward normal on the Neumann boundary $\Gamma_{N}^{f}, q$ is the source or sink term, $\mathbf{K}$ is the uniformly symmetric positive definite absolute permeability tensor, $\mu$ is the fluid viscosity, $\rho_{0}$ is a reference density, $\kappa=\frac{K}{\mu}$ is a measure of the hydraulic conductivity of the pore fluid, $c$ is the fluid compressibility, $T>0$ is the time interval, $\alpha$ is the Biot constant and $M \equiv\left(\phi_{0} c+\frac{\left(\alpha-\phi_{0}\right)(1-\alpha)}{K_{b}}\right)^{-1}$ is the Biot modulus with $K_{b}$ being the drained bulk modulus.

\section{POROMECHANICS MODEL}

Let $\Omega \subset \mathbb{R}^{3}$ be the poromechanics domain with boundary $\partial \Omega=\Gamma_{D}^{p} \cup \Gamma_{N}^{p}$ where $\Gamma_{D}^{p}$ is Dirichlet boundary and $\Gamma_{N}^{p}$ is Neumann boundary. The linear momentum balance in the quasi-static limit of interest (6) with the definition of the total stress (7) (see [30]) with the expression for the body force (8) and the small strain assumption (9) with boundary conditions (10) and initial condition (11) is

$$
\begin{aligned}
& \nabla \cdot \boldsymbol{\sigma}+\mathbf{f}=0 \\
& \boldsymbol{\sigma}=\mathbb{D} \mathbf{\epsilon}-\alpha p \mathbf{I} \equiv \lambda \bar{\epsilon} \mathbf{I}+2 G \mathbf{\epsilon}-\alpha p \mathbf{I} \\
& \mathbf{f}=\rho \phi \mathbf{g}+\rho_{r}(1-\phi) \mathbf{g} \\
& \mathbf{\epsilon}(\mathbf{u})=\frac{1}{2}\left(\nabla \mathbf{u}+\nabla^{T} \mathbf{u}\right) \\
& \mathbf{u} \cdot \mathbf{n}_{1}=0 \text { on } \Gamma_{D}^{p} \times[0, T], \boldsymbol{\sigma}^{T} \mathbf{n}_{2}=\mathbf{t} \text { on } \Gamma_{N}^{p} \times[0, T] \\
& \mathbf{u}(\mathbf{x}, 0)=0 \quad \forall \mathbf{x} \in \Omega
\end{aligned}
$$

where $\mathbf{u}: \Omega \times[0, T] \rightarrow \mathbb{R}^{3}$ is the solid displacement, $\rho_{r}$ is the rock density, $G$ is the shear modulus, $\lambda$ is the Lame parameter, $\mathbf{n}_{1}$ is the unit outward normal to $\Gamma_{D}^{p}, \mathbf{n}_{2}$ is the unit outward normal to $\Gamma_{N}^{p}, \alpha$ is the Biot parameter,f is body force per unit volume, $\mathbf{t}$ is the traction boundary condition, $\boldsymbol{\epsilon}$ is the strain tensor, $\mathbb{D}$ is the fourth order elasticity tensor and $\mathbf{I}$ is the second order identity tensor. The generalized Hooke's law (see [34])

$$
\sigma=\mathbb{M} \epsilon-\alpha p
$$


where $\mathbb{M}$ is the fourth order anisotropic elasticity tensor and $\alpha$ is the Biot tensor. The inverse of the generalized Hooke's law (12) is given by (see [37])

$$
\boldsymbol{\epsilon}=\mathbb{C} \boldsymbol{\sigma}+\frac{1}{3} C \mathbf{B} p
$$

where $\mathbb{C}$ is the fourth order anisotropic compliance tensor, $C(>0)$ is a generalized Hooke's law constant and $\mathbf{B}$ is a generalization of the Skempton pore pressure coefficient $B$ (see [38]) for anisotropic poroelasticity.

\section{FLUID CONTENT}

The fluid content $\zeta$ is given by (see [37])

$$
\zeta=C p+\frac{1}{3} C \mathbf{B}: \boldsymbol{\sigma} \equiv \frac{1}{M} p+\alpha: \boldsymbol{\epsilon}
$$

where $M(>0)$ is a generalization of the Biot modulus (see [39]) for anisotropic poroelasticity.

\section{STATEMENT OF CONTRACTION OF THE FIXED STRESS SPLIT SCHEME FOR ANISOTROPIC POROELASTICITY WITH BIOT TENSOR}

Let $\mathcal{T}_{h}$ be finite element partition of $\Omega$ consisting of distorted hexahedral elements $E$ where $h=\max _{E \in \mathcal{T}_{h}} \operatorname{diam}(E)$.

\subsection{Discrete variational statements for the flow subproblem}

Before arriving at the discrete variational statement of the flow model, we impose the fixed stress constraint on the strong form of the mass conservation equation (1). In lieu of (14), we write (1) as

$$
\begin{aligned}
& \frac{\partial}{\partial t}\left(C p+\frac{C}{3} \mathbf{B}: \boldsymbol{\sigma}\right)+\nabla \cdot \mathbf{z}=q \\
& C \frac{\partial p}{\partial t}+\nabla \cdot \mathbf{z}=q-\frac{C}{3} \mathbf{B}: \frac{\partial \boldsymbol{\sigma}}{\partial t}
\end{aligned}
$$

Using backward Euler in time, the discrete in time form of (15) for the $m^{\text {th }}$ coupling iteration in the $(n+1)^{t h}$ time step is written as

$$
C \frac{1}{\Delta t}\left(p^{m, n+1}-p^{n}\right)+\nabla \cdot \mathbf{z}^{m, n+1}=q^{n+1}-\frac{1}{\Delta t} \frac{C}{3} \mathbf{B}:\left(\boldsymbol{\sigma}^{m, n+1}-\boldsymbol{\sigma}^{n}\right)
$$

where $\Delta t$ is the time step and the source term as well as the terms evaluated at the previous time level $n$ do not depend on the coupling iteration count as they are known quantities. The fixed stress constraint implies that $\boldsymbol{\sigma}^{m, n+1}$ gets replaced by $\boldsymbol{\sigma}^{m-1, n+1}$ i.e. the computation of $p^{m, n+1}$ and $\mathbf{z}^{m, n+1}$ is based on the value of stress updated after the poromechanics solve from the previous coupling iteration $m-1$ at the current time level $n+1$. The modified equation is written as

$$
C\left(p^{m, n+1}-p^{n}\right)+\Delta t \nabla \cdot \mathbf{z}^{m, n+1}=\Delta t q^{n+1}-\frac{C}{3} \mathbf{B}:\left(\boldsymbol{\sigma}^{m, n+1}-\boldsymbol{\sigma}^{n}\right)
$$

As a result, the discrete weak form of (1) is given by

$$
\begin{aligned}
& C\left(p_{h}^{m, n+1}-p_{h}^{n}, \theta_{h}\right)_{\Omega}+\Delta t\left(\nabla \cdot \mathbf{z}_{h}^{m, n+1}, \theta_{h}\right)_{\Omega} \\
& =\Delta t\left(q^{n+1}, \theta_{h}\right)_{\Omega}-\frac{C}{3}\left(\mathbf{B}:\left(\boldsymbol{\sigma}^{m-1, n+1}-\boldsymbol{\sigma}^{n}\right), \theta_{h}\right)_{\Omega}
\end{aligned}
$$


Replacing $m$ by $m+1$ and subtracting the two equations, we get

$$
C\left(\delta^{(m)} p_{h}, \theta_{h}\right)_{\Omega}+\Delta t\left(\nabla \cdot \delta^{(m)} \mathbf{z}_{h}, \theta_{h}\right)_{\Omega}=-\frac{C}{3}\left(\mathbf{B}: \delta^{(m-1)} \boldsymbol{\sigma}, \theta_{h}\right)_{\Omega}
$$

The weak form of the Darcy law (2) for the $m^{\text {th }}$ coupling iteration in the $(n+1)^{t h}$ time step is given by

$$
\left(\boldsymbol{\kappa}^{-1} \mathbf{z}^{m, n+1}, \mathbf{v}\right)_{\Omega}=-\left(\nabla p^{m, n+1}, \mathbf{v}\right)_{\Omega}+\left(\rho_{0} \mathbf{g}, \mathbf{v}\right)_{\Omega} \forall \mathbf{v} \in \mathbf{V}(\Omega)
$$

where $\mathbf{V}(\Omega)$ is given by

$$
\mathbf{V}(\Omega) \equiv \mathbf{H}(\operatorname{div}, \Omega) \cap\left\{\mathbf{v}: \mathbf{v} \cdot \mathbf{n}=0 \text { on } \Gamma_{N}^{f}\right\}
$$

We use the divergence theorem to evaluate the first term on RHS of (16) as follows

$$
\begin{aligned}
& \left(\nabla p^{m, n+1}, \mathbf{v}\right)_{\Omega}=\left(\nabla, p^{m, n+1} \mathbf{v}\right)_{\Omega}-\left(p^{m, n+1}, \nabla \cdot \mathbf{v}\right)_{\Omega} \\
& =\left(p^{m, n+1}, \mathbf{v} \cdot \mathbf{n}\right)_{\partial \Omega}-\left(p^{m, n+1}, \nabla \cdot \mathbf{v}\right)_{\Omega} \\
& =(g, \mathbf{v} \cdot \mathbf{n})_{\Gamma_{D}^{f}}-\left(p^{m, n+1}, \nabla \cdot \mathbf{v}\right)_{\Omega}
\end{aligned}
$$

where we invoke $\mathbf{v} \cdot \mathbf{n}=0$ on $\Gamma_{N}^{f}$. In lieu of (16) and (17), we get

$$
\left(\boldsymbol{\kappa}^{-1} \mathbf{z}^{m, n+1}, \mathbf{v}\right)_{\Omega}=-(g, \mathbf{v} \cdot \mathbf{n})_{\Gamma_{D}^{f}}+\left(p^{m, n+1}, \nabla \cdot \mathbf{v}\right)_{\Omega}+\left(\rho_{0} \mathbf{g}, \mathbf{v}\right)_{\Omega}
$$

Replacing $m$ by $m+1$ and subtracting the two equations, we get

$$
\left(\boldsymbol{\kappa}^{-1} \delta^{(m)} \mathbf{z}_{h}, \mathbf{v}_{h}\right)_{\Omega}=\left(\delta^{(m)} p_{h}, \nabla \cdot \mathbf{v}_{h}\right)_{\Omega}
$$

\subsection{Discrete variational statement for the poromechanics subproblem}

The weak form of the linear momentum balance (6) is given by

$$
(\nabla \cdot \boldsymbol{\sigma}, \mathbf{q})_{\Omega}+(\mathbf{f} \cdot \mathbf{q})_{\Omega}=0 \quad(\forall \mathbf{q} \in \mathbf{U}(\Omega))
$$

where $\mathbf{U}(\Omega)$ is given by

$$
\mathbf{U}(\Omega) \equiv\left\{\mathbf{q}=(u, v, w): u, v, w \in H^{1}(\Omega), \mathbf{q}=0 \text { on } \Gamma_{D}^{p}\right\}
$$

We know from tensor calculus that

$$
(\nabla \cdot \boldsymbol{\sigma}, \mathbf{q})_{\Omega} \equiv(\nabla, \boldsymbol{\sigma q})_{\Omega}-(\boldsymbol{\sigma}: \nabla \mathbf{q})_{\Omega}
$$

Further, using the divergence theorem and the symmetry of $\boldsymbol{\sigma}$, we arrive at

$$
(\nabla, \boldsymbol{\sigma q})_{\Omega} \equiv(\mathbf{q}, \boldsymbol{\sigma n})_{\partial \Omega}
$$

We decompose $\nabla \mathbf{q}$ into a symmetric part $(\nabla \mathbf{q})_{s} \equiv \frac{1}{2}\left(\nabla \mathbf{q}+(\nabla \mathbf{q})^{T}\right) \equiv \mathbf{\epsilon}(\mathbf{q})$ and skew-symmetric part $(\nabla \mathbf{q})_{s s}$ and note that the contraction between a symmetric and skew-symmetric tensor is zero to obtain

$$
\boldsymbol{\sigma}: \nabla \mathbf{q} \equiv \boldsymbol{\sigma}:(\nabla \mathbf{q})_{s}+0 \boldsymbol{\sigma}:(\nabla \mathbf{q})_{s s}=\boldsymbol{\sigma}: \mathbf{\epsilon}(\mathbf{q})
$$

From (18), (19), (20) and (21), we get

$$
(\boldsymbol{\sigma n}, \mathbf{q})_{\partial \Omega}-(\boldsymbol{\sigma}: \mathbf{\epsilon}(\mathbf{q}))_{\Omega}+(\mathbf{f}, \mathbf{q})_{\Omega}=0
$$

which, after invoking the traction boundary condition, results in the discrete weak form for the $m^{\text {th }}$ coupling iteration as

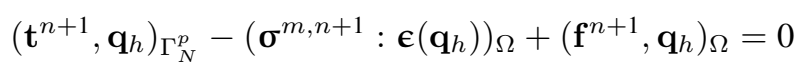

Replacing $m$ by $m+1$ and subtracting the two equations, we get

$$
\left(\delta^{(m)} \boldsymbol{\sigma}: \boldsymbol{\epsilon}\left(\mathbf{q}_{h}\right)\right)_{\Omega}=0
$$




\subsection{Summary of discrete variational statements in terms of coupling iteration differences}

The discrete variational statements in terms of coupling iteration differences is : find $\delta^{(m)} p_{h} \in W_{h}, \delta^{(m)} \mathbf{z}_{h} \in \mathbf{V}_{h}$ and $\delta^{(m)} \mathbf{u}_{h} \in \mathbf{U}_{h}$ such that

$$
\begin{aligned}
& C\left(\delta^{(m)} p_{h}, \theta_{h}\right)_{\Omega}+\Delta t\left(\nabla \cdot \delta^{(m)} \mathbf{z}_{h}, \theta_{h}\right)_{\Omega}=-\frac{C}{3}\left(\mathbf{B}: \delta^{(m-1)} \boldsymbol{\sigma}, \theta_{h}\right)_{\Omega} \\
& \left(\boldsymbol{\kappa}^{-1} \delta^{(m)} \mathbf{z}_{h}, \mathbf{v}_{h}\right)_{\Omega}=\left(\delta^{(m)} p_{h}, \nabla \cdot \mathbf{v}_{h}\right)_{\Omega} \\
& \left(\delta^{(m)} \boldsymbol{\sigma}: \boldsymbol{\epsilon}\left(\mathbf{q}_{h}\right)\right)_{\Omega}=0
\end{aligned}
$$

where the finite dimensional spaces $W_{h}, \mathbf{V}_{h}$ and $\mathbf{U}_{h}$ are

$$
\begin{aligned}
& W_{h}=\left\{\theta_{h}: \theta_{h} \mid \in P_{0}(E) \forall E \in T_{h}\right\} \\
& \mathbf{V}_{h}=\left\{\mathbf{v}_{h}:\left.\left.\mathbf{v}_{h}\right|_{E} \leftrightarrow \hat{\mathbf{v}}\right|_{\hat{E}} \in \hat{\mathbf{V}}(\hat{E}) \forall E \in T_{h}, \mathbf{v}_{h} \cdot \mathbf{n}=0 \text { on } \Gamma_{N}^{f}\right\} \\
& \mathbf{U}_{h}=\left\{\mathbf{q}_{h}=\left.(u, v, w)\right|_{E} \in Q_{1}(E) \forall E \in T_{h}, \mathbf{q}_{h}=0 \text { on } \Gamma_{D}^{p}\right\}
\end{aligned}
$$

Theorem 1. The fixed stress split iterative coupling scheme for anisotropic poroelasticity with Biot tensor in which the flow problem is solved first by freezing all components of the stress tensor is a contraction given by

$$
\begin{aligned}
& \left\|\mathbf{B}: \delta^{(m)} \boldsymbol{\sigma}\right\|_{\Omega}^{2}+\overbrace{2\left\|\delta^{(m)} p_{h}\right\|_{\Omega}^{2}}^{>0}+\overbrace{\frac{6 \Delta t}{C}\left\|\boldsymbol{\kappa}^{-1 / 2} \delta^{(m)} \mathbf{z}_{h}\right\|_{\Omega}^{2}}^{>0}+\overbrace{\frac{6}{C}\left(\delta^{(m)} \boldsymbol{\sigma}: \mathbb{C} \delta^{(m)} \boldsymbol{\sigma}\right)_{\Omega}}^{\geq 0} \\
& +\overbrace{\frac{3}{C^{2}\left\|\delta^{(m)} \zeta\right\|_{\Omega}^{2}}}^{>0}-\frac{6}{C^{2}}\left\|\delta^{(m)} \zeta-\delta_{f}^{(m)} \zeta\right\|_{\Omega}^{2} \leq\left\|\mathbf{B}: \delta^{(m-1)} \boldsymbol{\sigma}\right\|_{\Omega}^{2}
\end{aligned}
$$

where the quantity $\left\|\delta^{(m)} \zeta-\delta_{f}^{(m)} \zeta\right\|_{\Omega}^{2}$ is driven to a small value via the convergence criterion for the algorithm.

\section{Proof. • Step 1: Flow equations}

Testing (22) with $\theta_{h} \equiv \delta^{(m)} p_{h}$, we get

$$
C\left\|\delta^{(m)} p_{h}\right\|_{\Omega}^{2}+\Delta t\left(\nabla \cdot \delta^{(m)} \mathbf{z}_{h}, \delta^{(m)} p_{h}\right)_{\Omega}=-\frac{C}{3}\left(\mathbf{B}: \delta^{(m-1)} \boldsymbol{\sigma}, \delta^{(m)} p_{h}\right)_{\Omega}
$$

Testing (23) with $\mathbf{v}_{h} \equiv \delta^{(m)} \mathbf{z}_{h}$, we get

$$
\left\|\boldsymbol{\kappa}^{-1 / 2} \delta^{(m)} \mathbf{z}_{h}\right\|_{\Omega}^{2}=\left(\delta^{(m)} p_{h}, \nabla \cdot \delta^{(m)} \mathbf{z}_{h}\right)_{\Omega}
$$

From (25) and (26), we get

$$
C\left\|\delta^{(m)} p_{h}\right\|_{\Omega}^{2}+\Delta t\left\|\boldsymbol{\kappa}^{-1 / 2} \delta^{(m)} \mathbf{z}_{h}\right\|_{\Omega}^{2}=-\frac{C}{3}\left(\mathbf{B}: \delta^{(m-1)} \boldsymbol{\sigma}, \delta^{(m)} p_{h}\right)_{\Omega}
$$

- Step 2: Poromechanics equations

Testing (24) with $\mathbf{q}_{h} \equiv \delta^{(m)} \mathbf{u}_{h}$, we get

$$
\left(\delta^{(m)} \boldsymbol{\sigma}: \delta^{(m)} \boldsymbol{\epsilon}\right)_{\Omega}=0
$$

We now invoke (13) to arrive at the expression for change in strain tensor over the $(m+1)^{\text {th }}$ coupling iteration as follows

$$
\delta^{(m)} \boldsymbol{\epsilon}=\mathbb{C} \delta^{(m)} \boldsymbol{\sigma}+\frac{C}{3} \mathbf{B} \delta^{(m)} p_{h}
$$


Substituting (29) in (28), we get

$$
\left(\delta^{(m)} \boldsymbol{\sigma}: \mathbb{C} \delta^{(m)} \boldsymbol{\sigma}\right)_{\Omega}+\frac{C}{3}\left(\mathbf{B}: \delta^{(m)} \boldsymbol{\sigma}, \delta^{(m)} p_{h}\right)_{\Omega}=0
$$

\section{- Step 3: Combining flow and poromechanics equations}

Adding (27) and (30), we get

$$
\begin{aligned}
& C\left\|\delta^{(m)} p_{h}\right\|_{\Omega}^{2}+\Delta t\left\|\boldsymbol{\kappa}^{-1 / 2} \delta^{(m)} \mathbf{z}_{h}\right\|_{\Omega}^{2}+\left(\delta^{(m)} \boldsymbol{\sigma}: \mathbb{C} \delta^{(m)} \boldsymbol{\sigma}\right)_{\Omega} \\
& +\frac{C}{3}\left(\mathbf{B}: \delta^{(m)} \boldsymbol{\sigma}, \delta^{(m)} p_{h}\right)_{\Omega}=-\frac{C}{3}\left(\mathbf{B}: \delta^{(m-1)} \boldsymbol{\sigma}, \delta^{(m)} p_{h}\right)_{\Omega}
\end{aligned}
$$

\section{- Step 4: Variation in fluid content}

In lieu of (14), the variation in fluid content in the $(m+1)^{t h}$ coupling iteration is

$$
\delta^{(m)} \zeta=C \delta^{(m)} p_{h}+\frac{C}{3} \mathbf{B}: \delta^{(m)} \boldsymbol{\sigma}
$$

As a result, we can write

$$
\frac{1}{2 C}\left\|\delta^{(m)} \zeta\right\|_{\Omega}^{2}-\frac{C}{2}\left\|\delta^{(m)} p_{h}\right\|_{\Omega}^{2}-\frac{C}{18}\left\|\mathbf{B}: \delta^{(m)} \boldsymbol{\sigma}\right\|_{\Omega}^{2}=\frac{C}{3}\left(\mathbf{B}: \delta^{(m)} \boldsymbol{\sigma}, \delta^{(m)} p_{h}\right)_{\Omega}
$$

From (31) and (33), we get

$$
\begin{gathered}
C\left\|\delta^{(m)} p_{h}\right\|_{\Omega}^{2}+\Delta t\left\|\boldsymbol{\kappa}^{-1 / 2} \delta^{(m)} \mathbf{z}_{h}\right\|_{\Omega}^{2}+\left(\delta^{(m)} \boldsymbol{\sigma}: \mathbb{C} \delta^{(m)} \boldsymbol{\sigma}\right)_{\Omega}+\frac{1}{2 C}\left\|\delta^{(m)} \zeta\right\|_{\Omega}^{2} \\
-\frac{C}{2}\left\|\delta^{(m)} p_{h}\right\|_{\Omega}^{2}-\frac{C}{18}\left\|\mathbf{B}: \delta^{(m)} \boldsymbol{\sigma}\right\|_{\Omega}^{2}=-\frac{C}{3}\left(\mathbf{B}: \delta^{(m-1)} \boldsymbol{\sigma}, \delta^{(m)} p_{h}\right)_{\Omega}
\end{gathered}
$$

Adding and subtracting $\frac{C}{6}\left\|\mathbf{B}: \delta^{(m)} \boldsymbol{\sigma}\right\|_{\Omega}^{2}$ to the LHS of (34) results in

$$
\begin{aligned}
& \frac{C}{6}\left\|\mathbf{B}: \delta^{(m)} \boldsymbol{\sigma}\right\|_{\Omega}^{2}+\frac{C}{2}\left\|\delta^{(m)} p_{h}\right\|_{\Omega}^{2}+\Delta t\left\|\boldsymbol{\kappa}^{-1 / 2} \delta^{(m)} \mathbf{z}_{h}\right\|_{\Omega}^{2}+\left(\delta^{(m)} \boldsymbol{\sigma}: \mathbb{C} \delta^{(m)} \boldsymbol{\sigma}\right)_{\Omega} \\
& +\frac{1}{2 C}\left\|\delta^{(m)} \zeta\right\|_{\Omega}^{2}-\frac{C}{9}\left\|\mathbf{B}: \delta^{(m)} \boldsymbol{\sigma}\right\|_{\Omega}^{2}=-\frac{C}{3}\left(\mathbf{B}: \delta^{(m-1)} \boldsymbol{\sigma}, \delta^{(m)} p_{h}\right)_{\Omega}
\end{aligned}
$$

In lieu of (14) and the fixed stress constraint during the flow solve, the variation in fluid content during the flow solve in the $(m+1)^{t h}$ coupling iteration is given by

$$
\delta_{f}^{(m)} \zeta=C \delta_{f}^{(m)} p_{h}+\frac{C}{3} \mathbf{B}: 0 \delta_{f}^{(m)} \boldsymbol{\sigma}
$$

Further, since the pore pressure is frozen during the poromechanical solve, we have $\delta_{f}^{(m)} p_{h}=\delta^{(m)} p_{h}$. As a result, we can write

$$
\delta_{f}^{(m)} \zeta=C \delta^{(m)} p_{h}
$$

Subtracting (36) from (32), we can write

$$
\delta^{(m)} \zeta-\delta_{f}^{(m)} \zeta=\frac{C}{3} \mathbf{B}: \delta^{(m)} \boldsymbol{\sigma}
$$

which implies that

$$
\frac{1}{C}\left\|\delta^{(m)} \zeta-\delta_{f}^{(m)} \zeta\right\|_{\Omega}^{2}=\frac{C}{9}\left\|\mathbf{B}: \delta^{(m)} \boldsymbol{\sigma}\right\|_{\Omega}^{2}
$$


In lieu of (37), we can write (35) as

$$
\begin{aligned}
& \frac{C}{6}\left\|\mathbf{B}: \delta^{(m)} \boldsymbol{\sigma}\right\|_{\Omega}^{2}+\frac{C}{2}\left\|\delta^{(m)} p_{h}\right\|_{\Omega}^{2}+\Delta t\left\|\boldsymbol{\kappa}^{-1 / 2} \delta^{(m)} \mathbf{z}_{h}\right\|_{\Omega}^{2}+\left(\delta^{(m)} \boldsymbol{\sigma}: \mathbb{C} \delta^{(m)} \boldsymbol{\sigma}\right)_{\Omega} \\
& +\frac{1}{2 C}\left\|\delta^{(m)} \zeta\right\|_{\Omega}^{2}-\frac{1}{C}\left\|\delta^{(m)} \zeta-\delta_{f}^{(m)} \zeta\right\|_{\Omega}^{2}=-\frac{C}{3}\left(\mathbf{B}: \delta^{(m-1)} \boldsymbol{\sigma}, \delta^{(m)} p_{h}\right)_{\Omega}
\end{aligned}
$$

\section{- Step 5: Invoking positive-semidefiniteness of the compliance tensor}

The fourth order anisotropic compliance tensor $\mathbb{C}$ is positive-semidefinite (see [36]) in the sense that it obeys $\mathbf{S}$ : $\mathbb{C S} \geq 0$ for all symmetric tensors $\mathbf{S}$. In lieu of the above, since the Cauchy stress tensor is symmetric, the following holds true

$$
\delta^{(m)} \boldsymbol{\sigma}: \mathbb{C} \delta^{(m)} \boldsymbol{\sigma} \geq 0
$$

In lieu of (39), we can write (38) as

$$
\begin{aligned}
& \frac{C}{6}\left\|\mathbf{B}: \delta^{(m)} \boldsymbol{\sigma}\right\|_{\Omega}^{2}+\overbrace{\frac{C}{2}\left\|\delta^{(m)} p_{h}\right\|_{\Omega}^{2}}^{>0}+\overbrace{\Delta t\left\|\boldsymbol{\kappa}^{-1 / 2} \delta^{(m)} \mathbf{z}_{h}\right\|_{\Omega}^{2}}^{>0}+\overbrace{\left(\delta^{(m)} \boldsymbol{\sigma}: \mathbb{C} \delta^{(m)} \boldsymbol{\sigma}\right)_{\Omega}}^{\geq 0} \geq \\
& +\overbrace{\frac{1}{2 C}\left\|\delta^{(m)} \zeta\right\|_{\Omega}^{2}}^{>0}-\frac{1}{C}\left\|\delta^{(m)} \zeta-\delta_{f}^{(m)} \zeta\right\|_{\Omega}^{2}=-\frac{C}{3}\left(\mathbf{B}: \delta^{(m-1)} \boldsymbol{\sigma}, \delta^{(m)} p_{h}\right)_{\Omega}
\end{aligned}
$$

The quantity $\left\|\delta^{(m)} \zeta-\delta_{f}^{(m)} \zeta\right\|_{\Omega}^{2}$ on the LHS of (40) is driven to a small value via the convergence criterion for the algorithm as explained in Section 6.

\section{- Step 6: Invoking the Young's inequality}

Since the sum of the terms on the LHS of (40) is nonnegative, the RHS is also nonnegative. We invoke the Young's inequality (see [40])

$$
|a b| \leq \frac{a^{2}}{2}+\frac{b^{2}}{2}
$$

for the RHS of (40) as follows

$$
-\frac{C}{3}\left(\mathbf{B}: \delta^{(m-1)} \boldsymbol{\sigma}, \delta^{(m)} p_{h}\right)_{\Omega} \leq \frac{C}{3}\left(\frac{1}{2}\left\|\mathbf{B}: \delta^{(m-1)} \boldsymbol{\sigma}\right\|_{\Omega}^{2}+\frac{1}{2}\left\|\delta^{(m)} p_{h}\right\|_{\Omega}^{2}\right)
$$

In lieu of (41), we write (40) as

$$
\begin{gathered}
\frac{C}{6}\left\|\mathbf{B}: \delta^{(m)} \boldsymbol{\sigma}\right\|_{\Omega}^{2}+\overbrace{\frac{C}{2}\left\|\delta^{(m)} p_{h}\right\|_{\Omega}^{2}}^{>0}+\overbrace{\Delta t\left\|\boldsymbol{\kappa}^{-1 / 2} \delta^{(m)} \mathbf{z}_{h}\right\|_{\Omega}^{2}}^{>0}+\overbrace{\left(\delta^{(m)} \boldsymbol{\sigma}: \mathbb{C} \delta^{(m)} \boldsymbol{\sigma}\right)_{\Omega}}^{>0} \\
+\overbrace{\frac{1}{2 C}\left\|\delta^{(m)} \zeta\right\|_{\Omega}^{2}}^{>0}-\frac{1}{C}\left\|\delta^{(m)} \zeta-\delta_{f}^{(m)} \zeta\right\|_{\Omega}^{2} \leq \frac{C}{6}\left\|\mathbf{B}: \delta^{(m-1)} \boldsymbol{\sigma}\right\|_{\Omega}^{2}+\frac{C}{6}\left\|\delta^{(m)} p_{h}\right\|_{\Omega}^{2}
\end{gathered}
$$

which can also be written as

$$
\begin{aligned}
& \left\|\mathbf{B}: \delta^{(m)} \boldsymbol{\sigma}\right\|_{\Omega}^{2}+\overbrace{2\left\|\delta^{(m)} p_{h}\right\|_{\Omega}^{2}}^{>0}+\overbrace{\frac{6 \Delta t}{C}\left\|\boldsymbol{\kappa}^{-1 / 2} \delta^{(m)} \mathbf{z}_{h}\right\|_{\Omega}^{2}}^{>0}+\overbrace{\frac{6}{C}\left(\delta^{(m)} \boldsymbol{\sigma}: \mathbb{C} \delta^{(m)} \boldsymbol{\sigma}\right)_{\Omega}}^{\geq 0} \\
& +\overbrace{\frac{3}{C^{2}\left\|\delta^{(m)} \zeta\right\|_{\Omega}^{2}}}^{>0}-\frac{6}{C^{2}\left\|\delta^{(m)} \zeta-\delta_{f}^{(m)} \zeta\right\|_{\Omega}^{2} \leq\left\|\mathbf{B}: \delta^{(m-1)} \boldsymbol{\sigma}\right\|_{\Omega}^{2}}
\end{aligned}
$$




\section{CONVERGENCE CRITERION}

In lieu of (14), the variation in fluid content $\delta_{f}^{(m)} \zeta$ measured during the flow solve in the $(m+1)^{t h}$ coupling iteration at any time step is

$$
\delta_{f}^{(m)} \zeta=\frac{1}{M} \delta_{f}^{(m)} p_{h}+\boldsymbol{\alpha}: \delta_{f}^{(m)} \boldsymbol{\epsilon}=\zeta_{p}^{m+1}-\zeta^{m}
$$

where $\zeta^{m}$ is the fluid content at the end of the previous or $m^{t h}$ coupling iteration and $\zeta_{p}^{m+1}$ serves as the predictor to the fluid content at the end of the current or $(m+1)^{t h}$ coupling iteration. Similarly, the variation in fluid content $\delta^{(m)} \zeta$ over the $(m+1)^{t h}$ coupling iteration (including the flow solve and poromechanics solve) at any time step is

$$
\delta^{(m)} \zeta=\frac{1}{M} \delta^{(m)} p_{h}+\alpha: \delta^{(m)} \boldsymbol{\epsilon}=\zeta_{c}^{m+1}-\zeta^{m}
$$

where $\zeta_{c}^{m+1}$ serves as the corrector to $\zeta_{p}^{m+1}$. Subtracting (43) from (44), we get

$$
\delta^{(m)} \zeta-\delta_{f}^{(m)} \zeta \equiv \zeta_{c}^{m+1}-\zeta_{p}^{m+1}=\alpha:\left(\delta^{(m)} \boldsymbol{\epsilon}-\delta_{f}^{(m)} \boldsymbol{\epsilon}\right)=\boldsymbol{\alpha}:\left(\boldsymbol{\epsilon}_{c}^{m+1}-\boldsymbol{\epsilon}_{p}^{m+1}\right)
$$

which implies that the difference between the predicted value and the corrected value of the fluid content at the end of the $(m+1)^{t h}$ coupling iteration is equal to the difference between the predicted value $\left(\mathbf{\epsilon}_{p}^{m+1}\right)$ and the corrected value $\left(\epsilon_{c}^{m+1}\right)$ of the strain tensor at the end of the $(m+1)^{t h}$ coupling iteration scaled by the Biot tensor $\alpha$.

In lieu of the above, the stopping criterion for coupling iterations at any time step is

$$
\left\|\frac{\delta^{(m)} \zeta-\delta_{f}^{(m)} \zeta}{\zeta_{c}^{m+1}}\right\|_{L^{\infty}} \equiv\left\|\frac{\zeta_{c}^{m+1}-\zeta_{p}^{m+1}}{\zeta_{c}^{m+1}}\right\|_{L^{\infty}} \leq T O L
$$

where $T O L>0$ is a pre-specified tolerance. We set $T O L=1 \times 10^{-8}$ for our simulations.

\section{CONTRACTED NOTATION, MATERIAL SYMMETRY AND TRANSVERSE ISOTROPY}

The generalized Hooke's law (12) written in indicial notation

$$
\sigma_{i j}=\mathbb{M}_{i j k l} \epsilon_{k l}-\alpha_{i j} p
$$

is rewritten in contracted notation as

$$
\sigma_{\beta}=\mathbb{M}_{\beta \gamma} \epsilon_{\gamma}-\alpha_{\beta} p
$$

where the transformation is accomplished by replacing the subscripts $i j$ (or $k l$ ) by $\beta$ (or $\gamma$ ) using the following rules

$\begin{array}{rlr}i j(\text { or } k l) & \longleftrightarrow & \beta(\text { or } \gamma) \\ 11 & \longleftrightarrow & 1 \\ 22 & \longleftrightarrow & 2 \\ 33 & \longleftrightarrow & 3 \\ 23(\text { or } 32) & \longleftrightarrow & 4 \\ 31(\text { or } 13) & \longleftrightarrow & 5 \\ 12(\text { or } 21) & \longleftrightarrow & 6\end{array}$

In other words, the stress and strain tensors are represented as

$$
\begin{aligned}
& \boldsymbol{\sigma}=\left\{\begin{array}{llllll}
\sigma_{x x} & \sigma_{y y} & \sigma_{z z} & \sigma_{y z} & \sigma_{x z} & \sigma_{x y}
\end{array}\right\}^{T}, \\
& \boldsymbol{\epsilon}=\left\{\begin{array}{llllll}
\epsilon_{x x} & \epsilon_{y y} & \epsilon_{z z} & \epsilon_{y z} & \epsilon_{x z} & \epsilon_{x y}
\end{array}\right\}^{T}
\end{aligned}
$$


A material is said to possess a symmetry with respect to an orthogonal transformation $\chi$ if the elasticity tensor $\mathbb{M}$ is invariant under the orthogonal transformation $\chi$ as follows (see [41])

$$
\mathbb{M}^{\prime}=\mathbf{K M} \mathbf{K}^{T} \equiv \mathbb{M}
$$

where $\mathbb{M}^{\prime}$ is the transformed elasticity tensor and $\mathbf{K}$ is given by

$$
\begin{aligned}
\mathbf{K} & =\left[\begin{array}{cccccc}
\chi_{11}^{2} & \chi_{12}^{2} & \chi_{13}^{2} & 2 \chi_{12} \chi_{13} & 2 \chi_{13} \chi_{11} & 2 \chi_{11} \chi_{12} \\
\chi_{21}^{2} & \chi_{22}^{2} & \chi_{23}^{2} & 2 \chi_{22} \chi_{23} & 2 \chi_{23} \chi_{21} & 2 \chi_{21} \chi_{22} \\
\chi_{31}^{2} & \chi_{32}^{2} & \chi_{33}^{2} & 2 \chi_{32} \chi_{33} & 2 \chi_{33} \chi_{31} & 2 \chi_{31} \chi_{32} \\
\chi_{21} \chi_{31} & \chi_{22} \chi_{32} & \chi_{23} \chi_{33} & & \\
\chi_{31} \chi_{11} & \chi_{32} \chi_{12} & \chi_{33} \chi_{13} & \mathbf{K}^{\prime} \\
\chi_{11} \chi_{21} & \chi_{12} \chi_{22} & \chi_{13} \chi_{23} &
\end{array}\right] \\
\mathbf{K}^{\prime}= & {\left[\begin{array}{llll}
\chi_{22} \chi_{33}+\chi_{23} \chi_{32} & \chi_{23} \chi_{31}+\chi_{21} \chi_{33} & \chi_{21} \chi_{32}+\chi_{22} \chi_{31} \\
\chi_{32} \chi_{13}+\chi_{33} \chi_{12} & \chi_{33} \chi_{11}+\chi_{31} \chi_{13} & \chi_{31} \chi_{12}+\chi_{32} \chi_{11} \\
\chi_{12} \chi_{23}+\chi_{13} \chi_{22} & \chi_{13} \chi_{21}+\chi_{11} \chi_{23} & \chi_{11} \chi_{22}+\chi_{12} \chi_{21}
\end{array}\right] }
\end{aligned}
$$

where $\chi$ given by

$$
\chi=\mathbf{I}-2 \mathbf{n n}^{T}
$$

is a reflection with respect to a plane whose normal is $\mathbf{n}$. The plane is also refered to as the plane of material symmetry. The symmetry planes can be one of the following three possibilities

1. $x=0$ plane and any plane that contains the $x$-axis

2. $y=0$ plane and any plane that contains the $y$-axis

3. $z=0$ plane and any plane that contains the $z$-axis

For the Mandel's problem (see Figure 3), the material symmetry planes are the second possibility ( $y=0$ plane and any plane that contains the $y$-axis). In lieu of that, the elasticity tensor $\mathbb{M}$ reduces to

$$
\left[\begin{array}{cccccc}
\mathbb{M}_{11} & \mathbb{M}_{12} & \mathbb{M}_{13} & 0 & 0 & 0 \\
\mathbb{M}_{12} & \mathbb{M}_{22} & \mathbb{M}_{12} & 0 & 0 & 0 \\
\mathbb{M}_{13} & \mathbb{M}_{12} & \mathbb{M}_{11} & 0 & 0 & 0 \\
0 & 0 & 0 & 2 \mathbb{M}_{44} & 0 & 0 \\
0 & 0 & 0 & 0 & \left(\mathbb{M}_{11}-\mathbb{M}_{13}\right) & 0 \\
0 & 0 & 0 & 0 & 0 & 2 \mathbb{M}_{44}
\end{array}\right]
$$

with five independent componenents $\mathbb{M}_{11}, \mathbb{M}_{12}, \mathbb{M}_{13}, \mathbb{M}_{22}$ and $\mathbb{M}_{44}$.

\section{MANDEL'S PROBLEM}

The algorithm is implemented in the in-house parallel reservoir simulator at the Center for Subsurface Modeling. The bevo3 supercomputer at the Institute for Computational Engineering and Sciences is employed for all the simulations. The analytical solution provided by [35] to the Mandel's problem for transversely isotropic poroelasticity with compressible fluid and solid components serves as a benchmark for validation of coupled flow and poroelasticity codes.

\subsection{Geometry}

As shown in Figure 3, an infinitely long rectangular specimen is sandwiched between rigid, frictionless plates. For the transverse isotropy, the $y$-axis is assigned as the axis of material symmetry, which implies that at every point of the specimen along the $x z$-plane, the mechanical properties are the same in all directions. The lateral sides are free from normal and shear stress and pore pressure. At $t=0^{+}$, a force intensity of $2 \mathrm{~F} \mathrm{~N} / \mathrm{m}$ is applied to the rigid plates. 


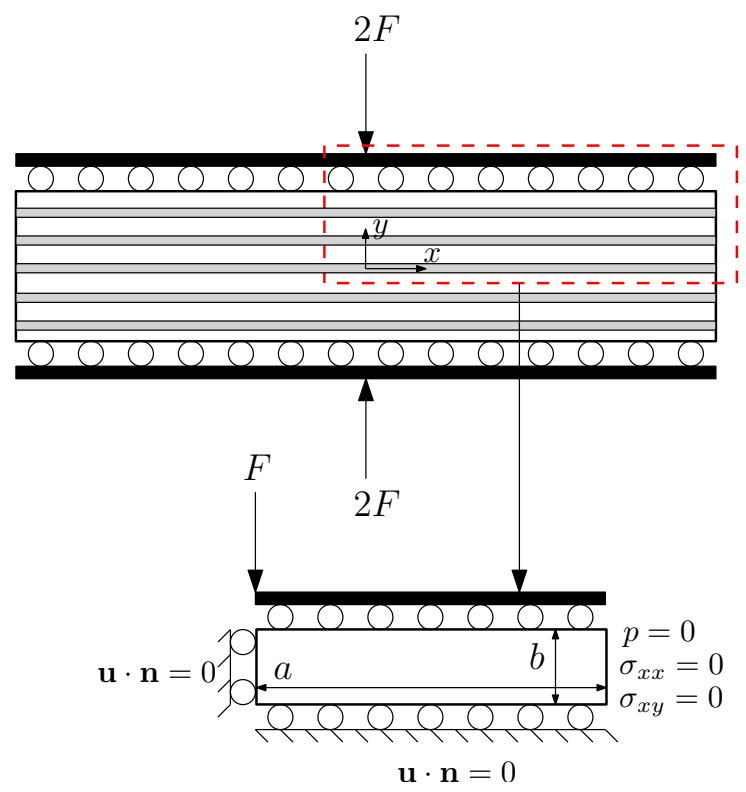

FIG. 3: Circles indicate rollers and solid black boxes indicate rigid frictionless plates. The material symmetry planes are the $y=0$ plane and any plane that contains the $y$-axis.

\subsection{Initial and boundary conditions}

The initial and boundary conditions are

$$
\begin{aligned}
& \left.\sigma_{x x}\right|_{t=0}=\left.\sigma_{x y}\right|_{t=0}=\left.\sigma_{y y}\right|_{t=0}=0,\left.p\right|_{t=0}=0 \\
& \left.\sigma_{x x}\right|_{x= \pm a}=\left.\sigma_{x y}\right|_{x= \pm a}=\left.\sigma_{y x}\right|_{y= \pm b}=\left.p\right|_{x= \pm a}=0 \\
& \int_{-a}^{a} \sigma_{y y} d x=-2 F, \mathbf{u} \cdot \mathbf{n}=0
\end{aligned}
$$$$
(\forall x, y)
$$$$
(\forall t, y= \pm b)
$$

where $\mathbf{n}$ is unit outward normal to the boundary. Plane strain condition is applicable i.e. $\epsilon_{z z}=0$. Given the biaxial symmetry of the problem, only a quarter of the domain needs to be modeled as shown in Figure 3. Following the approach of [42], the boundary conditions are recast as

$$
\begin{aligned}
& \left.\sigma_{x x}\right|_{x=a}=\left.\sigma_{x y}\right|_{x=a}=\left.\sigma_{y x}\right|_{y=b}=0,\left.\mathbf{u} \cdot \mathbf{n}\right|_{y=b}=U_{y} \\
& \left.\mathbf{u} \cdot \mathbf{n}\right|_{x=0}=\left.\mathbf{u} \cdot \mathbf{n}\right|_{y=0}=0,\left.p\right|_{x=a}=0 \\
& \left.\mathbf{u} \cdot \mathbf{n}\right|_{x=0}=\left.\mathbf{u} \cdot \mathbf{n}\right|_{y=0}=\left.\mathbf{u} \cdot \mathbf{n}\right|_{y=b}=0
\end{aligned}
$$

where $U_{y}$ represents the analytical solution for $u_{y}$ at $y=b$. 


\subsection{Stress-strain relations}

In lieu of (45), the stress-strain relations (with the plane strain assumption $\epsilon_{z z}=0$ and $\epsilon_{y z}=\epsilon_{x z}=0$ ) are

$$
\begin{aligned}
\left\{\begin{array}{l}
\sigma_{x x} \\
\sigma_{y y} \\
\sigma_{z z} \\
\sigma_{y z} \\
\sigma_{x z} \\
\sigma_{x y}
\end{array}\right\}= & {\left[\begin{array}{cccccc}
\mathbb{M}_{11} & \mathbb{M}_{12} & \mathbb{M}_{13} & 0 & 0 & 0 \\
\mathbb{M}_{12} & \mathbb{M}_{22} & \mathbb{M}_{12} & 0 & 0 & 0 \\
\mathbb{M}_{13} & \mathbb{M}_{12} & \mathbb{M}_{11} & 0 & 0 & 0 \\
0 & 0 & 0 & 2 \mathbb{M}_{44} & 0 & 0 \\
0 & 0 & 0 & 0 & 2 \mathbb{M}_{55} & 0 \\
0 & 0 & 0 & 0 & 0 & 2 \mathbb{M}_{44}
\end{array}\right]\left\{\begin{array}{c}
\epsilon_{x x} \\
\epsilon_{y y} \\
0 \epsilon_{z z} \\
0 \epsilon_{y z} \\
0 \epsilon_{x z} \\
\epsilon_{x y}
\end{array}\right\} } \\
& -\left[\begin{array}{cccccc}
\alpha_{x x} & 0 & 0 & 0 & 0 & 0 \\
0 & \alpha_{y y} & 0 & 0 & 0 & 0 \\
0 & 0 & \alpha_{x x} & 0 & 0 & 0 \\
0 & 0 & 0 & 0 & 0 & 0 \\
0 & 0 & 0 & 0 & 0 & 0 \\
0 & 0 & 0 & 0 & 0 & 0
\end{array}\right] p
\end{aligned}
$$

where $\mathbb{M}_{11}, \mathbb{M}_{12}, \mathbb{M}_{13}, \mathbb{M}_{22}, \mathbb{M}_{44}$ and $\mathbb{M}_{55}$ are given by (see [43])

$$
\begin{gathered}
\mathbb{M}_{11}=\frac{E_{x}\left(E_{y}-E_{x} v_{y x}^{2}\right)}{\left(1+v_{z x}\right)\left(E_{y}-E_{y} v_{z x}-2 E_{x} v_{y x}^{2}\right)} \\
\mathbb{M}_{12}=\frac{E_{x} E_{y} v_{y x}}{E_{y}-E_{y} v_{z x}-2 E_{x} v_{y x}^{2}} \\
\mathbb{M}_{13}=\frac{E_{x}\left(v_{z x} E_{y}+E_{x} v_{y x}^{2}\right)}{\left(1+v_{z x}\right)\left(E_{y}-E_{y} v_{z x}-2 E_{x} v_{y x}^{2}\right)} \\
\mathbb{M}_{22}=\frac{E_{y}^{2}\left(1-v_{z x}\right)}{E_{y}-E_{y} v_{z x}-2 E_{x} v_{y x}^{2}} \\
\mathbb{M}_{44}=G_{y z}=\frac{E_{y}}{2\left(1+v_{y x}\right)} \equiv \frac{E_{x}}{2\left(1+v_{x y}\right)} \\
\mathbb{M}_{55}=\left(\mathbb{M}_{11}-\mathbb{M}_{13}\right) / 2
\end{gathered}
$$

The set of data reported in [44] for Trafalgar shale are employed here

$$
E_{x}=20.6 G P a ; E_{y}=17.3 G P a ; v_{z x}=0.189 ; v_{y x}=0.246
$$

As a result, the five independent material parameters ((46a)-(46f)) are obtained as

$$
\begin{aligned}
& \mathbb{M}_{11}=24.1 G P a ; \mathbb{M}_{12}=7.62 G P a ; \mathbb{M}_{13}=6.8 G P a ; \\
& \mathbb{M}_{22}=21.0 G P a ; \mathbb{M}_{44}=6.94 G P a ; \mathbb{M}_{55}=8.66 G P a
\end{aligned}
$$

As a result, the elasticity matrix (see (45)) can be written as (all components in $G P a$ )

$$
\mathbb{M}=\left[\begin{array}{cccccc}
24.1 & 7.62 & 6.8 & 0 & 0 & 0 \\
7.62 & 21.0 & 7.62 & 0 & 0 & 0 \\
6.8 & 7.62 & 24.1 & 0 & 0 & 0 \\
0 & 0 & 0 & 13.88 & 0 & 0 \\
0 & 0 & 0 & 0 & 17.33 & 0 \\
0 & 0 & 0 & 0 & 0 & 13.88
\end{array}\right]
$$


The compliance tensor $\mathbb{C}$ is obtained as the inverse of the elasticity tensor $\mathbb{M}$ and is given by (all components in $\left.(G P a)^{-1}\right)$

$$
\mathbb{C}=\left[\begin{array}{cccccc}
0.0485 & -0.0142 & -0.0092 & 0 & 0 & 0 \\
-0.0142 & 0.05780 & -0.0142 & 0 & 0 & 0 \\
-0.0092 & -0.0142 & 0.0485 & 0 & 0 & 0 \\
0 & 0 & 0 & 0.07202 & 0 & 0 \\
0 & 0 & 0 & 0 & 0.05772 & 0 \\
0 & 0 & 0 & 0 & 0 & 0.07202
\end{array}\right]
$$

The specimen dimensions are taken to be $a \times b \equiv 100 \mathrm{~m} \times 10 \mathrm{~m}$ and the point load intensity is taken to be $10^{8} \mathrm{~N} / \mathrm{m}$. The initial porosity is taken to be 0.2 , the fluid compressibility is $3.03 \times 10^{-10} \mathrm{~Pa}^{-1}$, the $x$-permeability is $K_{x x}=$ $100 \mathrm{mD}$ and fluid viscosity is $\mu=0.001 \mathrm{~Pa}-\mathrm{s}$. Gravity is not active.

\subsection{Analytical solution}

The analytical solution $u_{x}, u_{y}$ and $p$ for the $x$-displacement, $y$-displacement and pressure respectively, are given by

$$
\begin{aligned}
u_{x}= & {\left[\frac{F}{a} \frac{\mathbb{M}_{12}}{\left(\mathbb{M}_{11} \mathbb{M}_{22}-\mathbb{M}_{12}^{2}\right)}-\frac{2 F}{a} \frac{\alpha_{x x} \alpha_{y y} M+\mathbb{M}_{12}}{A_{1} M\left(\alpha_{y y} \mathbb{M}_{11}-\alpha_{x x} \mathbb{M}_{12}\right)}\right.} \\
& \left.\times \sum_{i=1}^{\infty} \frac{\sin \beta_{i} \cos \beta_{i}}{\beta_{i}-\sin \beta_{i} \cos \beta_{i}} e^{-\beta_{i}^{2} c_{1} t / a^{2}}\right] x \\
& +\frac{2 F \alpha_{x x}}{A_{2} \mathbb{M}_{11}} \sum_{i=1}^{\infty} \frac{\cos \beta_{i} \sin \left(\beta_{i} x / a\right)}{\beta_{i}-\sin \beta_{i} \cos \beta_{i}} e^{-\beta_{i}^{2} c_{1} t / a^{2}} \\
u_{y}= & -\frac{F}{a} \frac{\mathbb{M}_{11}}{\mathbb{M}_{11} \mathbb{M}_{22}-\mathbb{M}_{12}^{2}} \\
& \times\left[1+2\left(A_{2} / A_{1}-1\right) \sum_{i=1}^{\infty} \frac{\sin \beta_{i} \cos \beta_{i}}{\beta_{i}-\sin \beta_{i} \cos \beta_{i}} e^{-\beta_{i}^{2} c_{1} t / a^{2}}\right] y \\
p= & \frac{2 F}{a A_{1}} \sum_{i=1}^{\infty} \frac{\sin \beta_{i}}{\beta_{i}-\sin \beta_{i} \cos \beta_{i}}\left(\cos \left(\beta_{i} x / a\right)-\cos \beta_{i}\right) e^{-\beta_{i}^{2} c_{1} t / a^{2}}
\end{aligned}
$$

\subsection{Micro-homogeneity and micro-isotropy}

The expressions (47) for $\alpha_{x x}$ and $\alpha_{y y}$ are obtained under the assumptions of micro-homogeneity and micro-isotropy. The micro-homogeneity assumption states that the skeleton of the porous material is homogeneous at the pore scale but can be heterogeneous at the macroscopic scale by having different micro-homogeneous material distributed in space ([37]). The micro-isotropy assumption states that the material is isotropic at the pore level such that it is characterized by the bulk modulus $K_{s}([45])$ and the macroscopic anisotropy is the manifestation of the directionality of the skeleton or the pore structure, and not the solid constituent itself.

$$
\alpha_{x x}=1-\frac{\mathbb{M}_{11}+\mathbb{M}_{12}+\mathbb{M}_{13}}{3 K_{s}} ; \alpha_{y y}=1-\frac{2 \mathbb{M}_{12}+\mathbb{M}_{22}}{3 K_{s}}
$$

Furthermore, the Biot modulus $M$ is given by

$$
M=\frac{K_{s}^{2}}{K_{s}\left(1+\phi_{0}\left(c K_{s}-1\right)\right)-\frac{2 \mathbb{M}_{11}+\mathbb{M}_{22}+2 \mathbb{M}_{13}+4 \mathbb{M}_{12}}{9}}
$$


while $\beta_{i}$ and $c_{1}$ are given as

$$
\begin{aligned}
A_{1} & =\frac{M\left(\alpha_{x x}^{2} \mathbb{M}_{22}-2 \alpha_{x x} \alpha_{y y} \mathbb{M}_{12}+\alpha_{y y}^{2} \mathbb{M}_{11}\right)+\mathbb{M}_{11} \mathbb{M}_{22}-\mathbb{M}_{12}^{2}}{M\left(\alpha_{y y} \mathbb{M}_{11}-\alpha_{x x} \mathbb{M}_{12}\right)} \\
A_{2} & =\frac{\alpha_{y y} \mathbb{M}_{11}-\alpha_{x x} \mathbb{M}_{12}}{\mathbb{M}_{11}} ; \frac{\tan \left(\beta_{i}\right)}{\beta_{i}}=\frac{A_{1}}{A_{2}} \\
c_{1} & =\frac{\kappa_{11} M \mathbb{M}_{11}}{\mathbb{M}_{11}+\alpha_{x x}^{2} M}
\end{aligned}
$$

where $K_{s}=48.2 G P a$ is the value as employed by [35]. As a result, we have

$$
\begin{aligned}
& \alpha_{x x}=0.733 ; \alpha_{y y}=0.749 ; M=13.93 G P a ; A_{1}=3.9050 ; \\
& A_{2}=0.5181 ; \frac{\tan \left(\beta_{i}\right)}{\beta_{i}}=7.5372 ; c_{1}=1.0486 \mathrm{~m}^{2} / \mathrm{s}
\end{aligned}
$$

Furthermore, the Hooke's law constant $C$ in (13) and (14) is given as

$$
C=2 \mathbb{C}_{11}+\mathbb{C}_{22}+2 \mathbb{C}_{13}+4 \mathbb{C}_{12}-\frac{1}{K_{s}}\left(1-\phi_{0}\left(c K_{s}-1\right)\right)=0.1154 \times 10^{-9} \mathrm{~Pa}^{-1}
$$

\subsection{Fluid content updates}

The fixed stress constraint $\delta_{f}^{(m)} \boldsymbol{\sigma}=0$ makes it convenient to measure $\delta_{f}^{(m)} \zeta$ using the definition of $\zeta$ in (14) which involves the pressure and stress as follows

$$
\delta_{f}^{(m)} \zeta \equiv \zeta_{p}^{m+1}-\zeta^{m}=C \delta_{f}^{(m)} p_{h}+\frac{C}{3} \mathbf{B}: 0 \delta_{f}^{(m)} \boldsymbol{\sigma}
$$

Since $\delta_{f}^{(m)} p_{h}$ is what we solve for in the flow system, $\delta_{f}^{(m)} \zeta$ can be easily measured using the above equation rather than (43). Since $\delta^{(m)} \boldsymbol{\epsilon}$ is obtained by post-processing the poromechanical solution, $\delta^{(m)} \zeta$ can be obtained using (44) as follows

$$
\delta^{(m)} \zeta \equiv \zeta_{c}^{m+1}-\zeta^{m}=\frac{1}{M} \delta^{(m)} p_{h}+\alpha: \delta^{(m)} \boldsymbol{\epsilon}=\frac{1}{M} \delta^{(m)} p_{h}+\alpha_{x x} \delta^{(m)} \epsilon_{x x}+\alpha_{y y} \delta^{(m)} \epsilon_{y y}
$$

\subsection{Optimal norm}

[46] have shown that when mixed finite element spaces are employed for fluxes and pressures and a continuous Galerkin space is employed for displacements, optimality should be achieved when the pressure solution error is measured in the $L^{\infty}\left(L^{2}\right)$ norm. Although the analysis of [46] stands for the case of isotropic poroelasticity with scalar Biot parameter, intuitively, we expect a similar result for the case of anisotropic poroelasticity with tensor Biot parameter. The error norm is computed using the midpoint quadrature rule:

$$
\left\|\frac{1}{M}\left(p-p_{h}\right)\right\|_{L^{\infty}\left(L^{2}\right)} \equiv \max _{0<\tau \leq T}\left(\sum_{E \in \mathcal{T}_{h}}|E|\left(\frac{p\left(\tau, m_{e}\right)-p_{h}\left(m_{e}\right)}{M}\right)^{2}\right)^{\frac{1}{2}}
$$

where $m_{e}$ is the center of mass of element $E$ and $T$ is the total time. To minimize the effects of the error produced by time discretization, a small time step of $1 \times 10^{-2} s$ is chosen. As shown in Table 1, we observe first order convergence for the pore pressure solution. 


\begin{tabular}{c|c|c}
\hline $\mathcal{T}_{h}$ & $\left\|\frac{1}{M}\left(p-p_{h}\right)\right\|_{L^{\infty}\left(L^{2}\right)}$ & Rate \\
\hline $10 \times 10$ & $0.2544 \times 10^{-1}$ & \\
\hline $20 \times 20$ & $0.1355 \times 10^{-1}$ & 0.9088 \\
\hline $30 \times 30$ & $0.8466 \times 10^{-2}$ & 1.1600 \\
\hline $40 \times 40$ & $0.6311 \times 10^{-2}$ & 1.0211 \\
\hline $50 \times 50$ & $0.5067 \times 10^{-2}$ & 0.9839 \\
\hline
\end{tabular}

TABLE 1: Order of convergence of pore pressure solution using the fixed stress split scheme for the Mandel's problem with transverse isotropy

\section{References}

[1] Hiroo Kanamori and Emily E Brodsky. The physics of earthquakes. Reports on Progress in Physics, 67(8):1429, 2004.

[2] Christopher H Scholz, Yen Joe Tan, and Fabien Albino. The mechanism of tidal triggering of earthquakes at mid-ocean ridges. Nature communications, 10(1):1-7, 2019.

[3] Sebastian Hainzl, Toni Kraft, Joachim Wassermann, Heiner Igel, and E Schmedes. Evidence for rainfall-triggered earthquake activity. Geophysical Research Letters, 33(19), 2006.

[4] EK Montgomery-Brown, David R Shelly, and Paul A Hsieh. Snowmelt-triggered earthquake swarms at the margin of long valley caldera, california. Geophysical Research Letters, 46(7):3698-3705, 2019.

[5] ChiChing Liu, Alan T Linde, and I Selwyn Sacks. Slow earthquakes triggered by typhoons. Nature, 459(7248):833-836, 2009.

[6] Gillian R Foulger, Miles P Wilson, Jon G Gluyas, Bruce R Julian, and Richard J Davies. Global review of human-induced earthquakes. Earth-Science Reviews, 178:438-514, 2018.

[7] Youssef MA Hashash, Jeffrey J Hook, Birger Schmidt, I John, and Chiang Yao. Seismic design and analysis of underground structures. Tunnelling and underground space technology, 16(4):247-293, 2001.

[8] Jack Moehle. Seismic design of reinforced concrete buildings. McGraw-Hill Education, 2015.

[9] Mark D Zoback and Steven M Gorelick. Earthquake triggering and large-scale geologic storage of carbon dioxide. Proceedings of the National Academy of Sciences, 109(26):10164-10168, 2012.

[10] William L Ellsworth, Domenico Giardini, John Townend, Shemin Ge, and Toshihiko Shimamoto. Triggering of the pohang, korea, earthquake ( $\mathrm{m}$ w 5.5) by enhanced geothermal system stimulation. Seismological Research Letters, 90(5):1844-1858, 2019.

[11] Katie M Keranen and Matthew Weingarten. Induced seismicity. Annual Review of Earth and Planetary Sciences, 46:149$174,2018$.

[12] Saumik Dana and Mary F Wheeler. Augmented lagrangian for treatment of hanging nodes in hexahedral meshes. arXiv preprint arXiv:1809.04031, 2018.

[13] Saumik Dana and Karthik Reddy Lyathakula. Uncertainty quantification in friction model for earthquakes using bayesian inference. arXiv preprint arXiv:2104.11156, 2021.

[14] S. Dana and M. F. Wheeler. Convergence analysis of fixed stress split iterative scheme for anisotropic poroelasticity with tensor biot parameter. Computational Geosciences, 22(5):1219-1230, 2018.

[15] S. Dana and M. F. Wheeler. Convergence analysis of two-grid fixed stress split iterative scheme for coupled flow and deformation in heterogeneous poroelastic media. Computer Methods in Applied Mechanics and Engineering, 341:788-806, 2018.

[16] Saumik Dana and Mary F Wheeler. Design of convergence criterion for fixed stress split iterative scheme for small strain anisotropic poroelastoplasticity coupled with single phase flow. arXiv preprint arXiv:1912.06476, 2019. 
[17] Saumik Dana and Mary F Wheeler. An efficient algorithm for numerical homogenization of fluid filled porous solids: part-i. arXiv preprint arXiv:2002.03770, 2020.

[18] Saumik Dana and Mary F Wheeler. A machine learning accelerated $\mathrm{fe}^{2}$ homogenization algorithm for elastic solids. arXiv preprint arXiv:2003.11372, 2020.

[19] Saumik Dana, Benjamin Ganis, and Mary F. Wheeler. A multiscale fixed stress split iterative scheme for coupled flow and poromechanics in deep subsurface reservoirs. Journal of Computational Physics, 352:1-22, 2018.

[20] Saumik Dana, Shriram Srinivasan, Satish Karra, Nataliia Makedonska, Jeffrey D Hyman, Daniel O’Malley, Hari Viswanathan, and Gowri Srinivasan. Towards real-time forecasting of natural gas production by harnessing graph theory for stochastic discrete fracture networks. Journal of Petroleum Science and Engineering, 195:107791, 2020.

[21] Saumik Dana, Joel Ita, and Mary F Wheeler. The correspondence between voigt and reuss bounds and the decoupling constraint in a two-grid staggered algorithm for consolidation in heterogeneous porous media. Multiscale Modeling \& Simulation, 18(1):221-239, 2020.

[22] Saumik Dana. A simple framework for arriving at bounds on effective moduli in heterogeneous anisotropic poroelastic solids. arXiv preprint arXiv: 1912.10835, 2019.

[23] Saumik Dana. System of equations and staggered solution algorithm for immiscible two-phase flow coupled with linear poromechanics. arXiv preprint arXiv:1912.04703, 2019.

[24] S. Dana. Addressing challenges in modeling of coupled flow and poromechanics in deep subsurface reservoirs. PhD thesis, The University of Texas at Austin, 2018.

[25] M. M. Carroll. An effective stress law for anisotropic elastic deformation. Journal of Geophysical Research, 84(B13):7510$7512,1979$.

[26] M. M. Carroll and N. Katsube. The role of terzaghi effective stress in linearly elastic deformation. Journal of Energy Resources Technology, 105(4):509-511, 1983.

[27] N. Katsube. The constitutive theory for fluid-filled porous materials. Journal of Applied Mechanics, 52(1):185-189, 1985.

[28] M. Thompson and J. R. Willis. A reformation of the equations of anisotropic poroelasticity. Journal of Applied Mechanics, 58(3):612-616, 1991.

[29] A. Nur and J. D. Byerlee. An exact effective stress law for elastic deformation of rock with fluids. Journal of Geophysical Research, 76(26):6414-6419, 1971.

[30] M. A. Biot. General theory of three dimensional consolidation. Journal of Applied Physics, 12:155-164, 1941.

[31] J. Geertsma. The effect of fluid pressure decline on volumetric changes of porous rocks. SPE, 210:331-340, 1957.

[32] A. W. Skempton. Significance of terzaghi's concept of effective stress (terzaghi's discovery of effective stress). In From theory to practice in soil mechanics (L. Bjerrum and A. Casagrande and R. B. Peek and A. W. Skempton, eds.). New YorkLondon: John Wiley and Sons 1960, 1960.

[33] M. A. Biot. Theory of elasticity and consolidation for a porous anisotropic solid. Journal of Applied Physics, 26(2):182-185, 1955.

[34] O. Coussy. Poromechanics. Wiley, 2nd ed edition, 2004.

[35] Y. Abousleiman, A. H. D. Cheng, L. Cui, E. Detournay, and J. C. Roegiers. Mandel's problem revisited. Géotechnique, 46(2):187-195, 1996.

[36] M. E. Gurtin, E. Fried, and L. Anand. The Mechanics and Thermodynamics of Continua. Cambridge University Press, 1 edition, 2010.

[37] A. H. D. Cheng. Material coefficients of anisotropic poroelasticity. International Journal of Rock Mechanics and Mining Sciences, 34:199-205, 1997. 
[38] A. W. Skempton. The pore-pressure coefficients a and b. Géotechnique, 4(4):143-147, 1954.

[39] M. A. Biot and D. G. Willis. The elastic coefficients of the theory of consolidation. Journal of Applied Mechanics, 24:594$601,1957$.

[40] J. M. Steele. The Cauchy-Schwarz Master Class: An Introduction to the Art of Mathematical Inequalities. Maa Problem Books Series. Cambridge University Press, 2004.

[41] T. C. T. Ting. Anisotropic elasticity: Theory and applications. The Oxford Engineering Science Series. Oxford University Press, 1996.

[42] A. Mikelić, B. Wang, and M. F. Wheeler. Numerical convergence study of iterative coupling for coupled flow and geomechanics. Computational Geosciences, 18(3):325-341, 2014.

[43] A. P. Boresi, K. P. Chong, and J. D. Lee. Elasticity in Engineering Mechanics. John Wiley and Sons, 3rd edition, 2010.

[44] T. Aoki, C. P. Tan, and W. E. Bamford. Effects of deformation and strength anisotropy on borehole failures in saturated shales. International Journal of Rock Mechanics and Mining Sciences and Geomechanics Abstracts, 30(7):1031-1034, 1993.

[45] L. Cui, A. H. D. Cheng, V. N. Kaliakin, Y. Abousleiman, and J. C. Roegiers. Finite element analyses of anisotropic poroelasticity: A generalized mandel's problem and an inclined borehole problem. International Journal for Numerical and Analytical Methods in Geomechanics, 20(6):381-401, 1996.

[46] P.J. Phillips and M.F. Wheeler. A coupling of mixed and continuous galerkin finite element methods for poroelasticity ii: the discrete-in-time case. Computational Geosciences, 11(2):145-158, 2007. 